\title{
Extraction and quantification of saponins: a review
}

\begin{abstract}
Saponins, a second metabolites mainly derived from plant materials, have been used extensively in drug-related industry due to the pharmaceutical properties. These have driven the emergence of various new extraction technologies with the main purpose to optimize the yield in order to accommodate the recent need. The plants containing saponins are discussed, and their pharmaceutical properties and applications in food are highlighted. This review focuses on the saponin extraction with emphasis on conventional and green technology techniques employed in previous works by relating to their specific objective in each study. The quantification methods of saponins yield, i.e., spectrophotometric and chromatographic, are summarized and discussed. In addition, this review aims to provide a point of reference to researchers who wish to design experiment to suit their particular objective in swift.
\end{abstract}

Keyword: Saponins; Conventional extraction; Green extraction technologies; Quantification; Spectrophotometric; Chromatographic 